\title{
Tradeoff between Area Spectral Efficiency and End-to-End Throughput in Rate-Adaptive Multihop Radio Networks
}

\author{
Koji YAMAMOTO $^{\dagger a)}$, Student Member and Susumu YOSHIDA ${ }^{\dagger}$, Fellow
}

\begin{abstract}
SUMMARY We investigate the impact of symbol rate control, modulation level control, and the number of hops on the area spectral efficiency of interference-limited multihop radio networks. By controlling symbol rate and modulation level, data rate can be adapted according to received power. In addition, varying the number of hops can control received power. First, we evaluate the achievable end-to-end throughput of multihop transmission assuming symbol rate and modulation level control. Numerical results reveal that by controlling symbol rate or using multihop transmission, the end-to-end communication range can be extended at the cost of end-to-end throughput, and this may result in lower area spectral efficiency. Next, an expression for the area spectral efficiency of multihop radio networks is derived as a function of the number of hops and the end-to-end throughput. Numerical results also reveal that the resulting area spectral efficiency depends on the specific circumstances, which, however, can be increased only by using multihop transmission.

key words: multihop radio networks, area spectral efficiency, bandwidth efficiency, rate adaptation
\end{abstract}

\section{Introduction}

Recent advancements in radio technologies and the success of the Internet have shown the potential of information systems as ubiquitous networks accessible from anywhere, at any time, and with any device [1]. A key enabling technology for such networks is wireless ad hoc networking. A key assumption is that any two nodes can communicate directly if there is an adequate radio propagation path between them subject to the maximum transmit power constraints of the nodes. Otherwise, packets are relayed from the source to the final destination, which can be relatively far apart, by multihop transmission [2].

Wireless ad hoc networks can become an information infrastructure formed by co-operating wireless nodes without centralized administration [3]. For this type of wide area radio networks, referred to as multihop radio networks, high system spectral efficiency is required since the amount of spectrum available for wireless systems is limited. This type of spectral efficiency, referred to as area spectral efficiency (ASE), is defined to be the maximum end-to-end bit rate through multiple hops per unit bandwidth per unit area as that defined in cellular networks [4]-[6]. Mukai et al. demonstrated that the ASE of multihop radio networks does not experience significant degradation compared with that of cellular networks [7].

\footnotetext{
Manuscript received January 4, 2005.

Manuscript revised April 8, 2005.

${ }^{\dagger}$ The authors are with the Graduate School of Informatics, Kyoto University, Kyoto-shi, 606-8501 Japan.

a) E-mail: kyamamot@hanase.kuee.kyoto-u.ac.jp DOI: 10.1093/ietcom/e88-b.9.3532
}

The ASE of multihop radio networks is sensitive to many factors, such as fairness of nodes [8]-[10], possible node mobility [11], and lack of centralized control [12], [13]. Gupta et al. estimated the throughput per sourcedestination pair in multihop radio networks with optimal routing and scheduling, and found that the smallest possible transmit power that still ensures connectivity may be most effective in terms of the ASE [14]. Toumpis et al. showed that if the data rate is adjusted to the received power, additional power control leads to negligible capacity gains in a wireless ad hoc network [15].

One of the advantages of multihop transmission over single-hop transmission is a larger end-to-end communication range. However, the spectral efficiency of multihop transmission is not necessarily the same as that of single-hop transmission. Therefore, to consider in what situation multihop transmission results in higher spectral efficiency may be of interest in system design. This type of spectral efficiency, referred to as bandwidth efficiency (BE), is defined to be the maximum end-to-end bit rate through multiple hops per unit bandwidth.

This paper presents a simple yet meaningful theoretical framework for analyzing the BE of multihop transmission and the ASE of interference-limited multihop radio networks. In our argument, we assume that the networks have controls for symbol rate and modulation level. The remainder of this paper is organized as follows. In Sect. 2, we discuss a system model. In Sect. 3, we consider a single isolated multihop route and evaluate the $\mathrm{BE}$ considering error accumulation over multihop relaying. In addition, we evaluate the effect of spatial channel reuse on the BE of multihop transmission in Sect. 4. In Sect. 5, we investigate the ASE of interference-limited multihop radio networks. Section 6 concludes the paper with a summary and some final remarks.

\section{System Model}

We assume that each node can be either transmitting or receiving at a given time, and omnidirectional antennas are used for the nodes. We assume propagation loss with the path loss exponent $\alpha$. The effects of shadowing and fast fading are not considered for the sake of simplicity, as has been done in [16]. All interference signals are assumed to be equivalent to additive white Gaussian noise over the channel (which is discussed in more detail in [5]). 
2.1 Bandwidth Efficiency of Symbol Rate and Modulation Level Control

We assume that the transmitting node adjusts the data rate $r$ based on the average received carrier-to-interference plus noise ratio (CINR) $\gamma$ for a given required bit error rate (BER) $p_{\mathrm{b}}$ as

$$
r=f_{1}\left(\gamma, p_{\mathrm{b}}\right) .
$$

Shannon capacity, which is the maximum of the information rates without error,

$$
f_{1}\left(\gamma, p_{\mathrm{b}}\right)=\log _{2}(1+\gamma),
$$

has been used to obtain the specific dependence of $r$ on $\gamma$ [13], [15], [17]. One of the problems of multihop transmission is accumulation of bit errors or packet errors over multihop relaying [18]; however, the bit error probability cannot be evaluated with Shannon capacity. Using results presented in [19], [20], we derived a specific dependence of $r$ on both $\gamma$ and $p_{\mathrm{b}}$ considering symbol rate control and modulation level control with $M$-ary quadrature amplitude modulation $(M$-QAM).

Let $\gamma_{\mathrm{QPSK}}$ denote the required CINR for QPSK (4QAM). When the received CINR is higher than $\gamma_{\mathrm{QPSK}}$, variable-rate $M$-QAM [19] ( $\leq M \leq 64)$ is used for modulation level control. As described in [19], the BE of this modulation level control in the additive white Gaussian noise channel is approximated by

$$
f_{1}\left(\gamma, p_{\mathrm{b}}\right)=\log _{2}\left(1+\beta_{1} \gamma\right), \quad \beta_{1}=-1.5 / \ln \left(5 p_{\mathrm{b}}\right),
$$

where the subscript of the $\operatorname{BE} f$ and that of the constant $\beta$ denote the number of hops. Since the BE of QPSK is 2, we have the required CINR for QPSK

$$
\gamma_{\mathrm{QPSK}}=3 / \beta_{1} \text {. }
$$

When the received CINR is lower than $\gamma_{\mathrm{QPSK}}$, the symbol rate is reduced to decrease the required CINR. Lower symbol rate transmission is equivalently obtained from consecutive transmission of the identical symbols [20]. When the symbol rate of QPSK is reduced to $1 / K(1<K \leq 4)$, the required CINR can be lowered by $10 \log _{10} K \mathrm{~dB}$ at the cost of $\mathrm{BE}$ as

$$
f_{1}\left(\gamma, p_{\mathrm{b}}\right)=2 / K .
$$

When $K$ is controlled to meet the received CINR $\gamma$, we have

$$
\gamma=\gamma_{\mathrm{QPSK}} / K .
$$

The approximated model for BE of symbol rate and modulation level control may be expressed by using Eqs. (3), (5), and (6),

$$
f_{1}\left(\gamma, p_{\mathrm{b}}\right)= \begin{cases}2 \beta_{1} \gamma / 3 & 3 / 4 \beta_{1} \leq \gamma<3 / \beta_{1} \\ \log _{2}\left(1+\beta_{1} \gamma\right) & 3 / \beta_{1} \leq \gamma \leq 63 / \beta_{1} .\end{cases}
$$

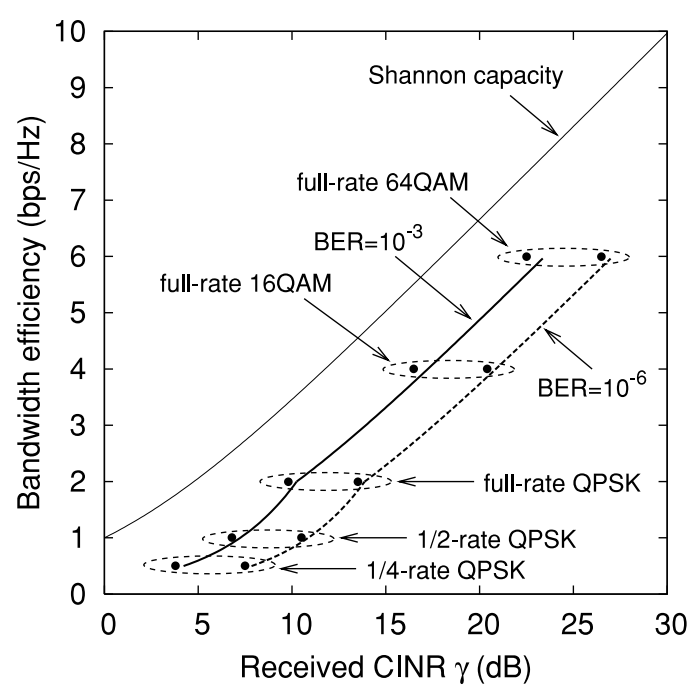

Fig.1 Bandwidth efficiency of symbol rate and modulation level control.

We compare the CINR dependence of the BE (7) at BER's of $10^{-3}$ and $10^{-6}$ in Fig. 1. Figure 1 also shows the bit error probability for coherent detection of 1/4-rate QPSK, 1/2-rate QPSK, QPSK, 16QAM, and 64QAM [21]. The required CINR of $M$-QAM is $5.5 \mathrm{~dB}\left(p_{\mathrm{b}}=10^{-3}\right)$ and $9.1 \mathrm{~dB}$ $\left(p_{\mathrm{b}}=10^{-6}\right)$ larger than that of Shannon capacity. When the symbol rate is reduced, the difference between the required CINR of this model and that of Shannon capacity becomes larger.

\section{Bandwidth Efficiency of Multihop Transmission without Spatial Channel Reuse}

\subsection{Analysis}

In this section, we evaluate the tradeoff between the achievable end-to-end throughput and the number of hops. Consider a single isolated source-destination pair. Under this environment, the CINR reduces to the carrier-to-noise ratio (CNR). Let the received CNR at the destination node be $\gamma$ as shown in Fig. 2(a), regardless of whether these two nodes can communicate directly (hereinafter referred to as "endto-end CNR"). End-to-end CNR is considered to be an appropriate parameter to reflect both the transmit power per node and the end-to-end distance since the absolute values of the transmit power and the distance do not have meaning in our simple model.

We assume regenerative relaying (sometimes referred to as digital relaying) such that relaying nodes decode and re-encode before retransmission. As shown in Fig. 2(b), we model the topology of $n$-hop transmission as equally spaced $(n-1)$ relaying nodes on a straight line segment from the source node to the destination node, and assume that spatial channel reuse is not allowed. While these assumptions are not necessarily realistic, they do enable us to gain insight into the design of multihop radio networks. We can switch from this single-hop transmission to $n$-hop transmission with the same transmit power and the same required 


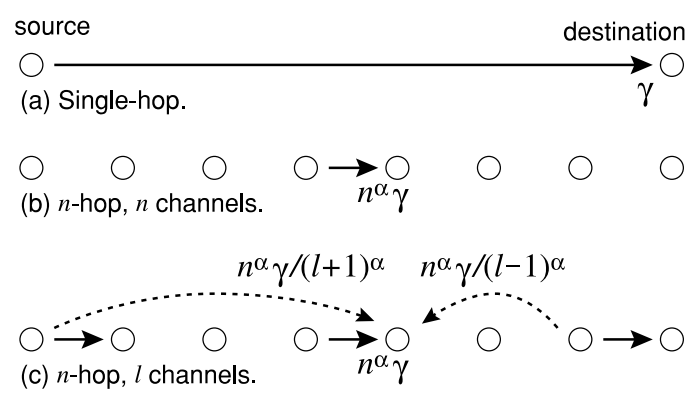

Fig. 2 Models of single-hop and multihop transmission $(n=7, \ell=3)$. Solid arrows represent desired signals and dashed arrows represent cochannel interference.

end-to-end BER $p_{\mathrm{b}}$ by allowing only one node at a time to transmit. Whether frequency division is used is immaterial to our results since time division and frequency division are equivalent in that they both divide up the spectrum orthogonally, and this orthogonal division results in the same number of channels [22], [23].

This switching has three effects. First, the per-hop distance is reduced to $1 / n$ of the end-to-end distance. Therefore, the per-hop CNR can be expressed as $n^{\alpha} \gamma$. This is one of the advantages of multihop transmission over single-hop transmission. Second, the required per-hop BER for $n$-hop transmission over an AWGN channel for a given required end-to-end BER $p_{\mathrm{b}}$ becomes $p_{\mathrm{b}} / n$. This is because when the required BER of every hop is $p_{\mathrm{h}}$, the end-to-end BER $p_{\mathrm{b}}$ becomes

$$
\begin{aligned}
p_{\mathrm{b}} & =1-\left(1-p_{\mathrm{h}}\right)^{n} \\
& \simeq n p_{\mathrm{h}} \quad\left(p_{\mathrm{h}} \ll 1\right) .
\end{aligned}
$$

Finally, the percentage of time a node can transmit a signal becomes approximately $1 / n$. Consequently, the BE of $n$ hop transmission at given end-to-end CNR $\gamma$ and required end-to-end BER $p_{\mathrm{b}}$ can be expressed as

$$
f_{n}\left(\gamma, p_{\mathrm{b}}\right)=\frac{1}{n} f_{1}\left(n^{\alpha} \gamma, p_{\mathrm{b}} / n\right)
$$

where $f_{1}$ is the $\mathrm{BE}$ of single-hop transmission (7). Therefore, this relationship between the BE of $n$-hop and that of single-hop is extendable when using other modulation schemes. The BE of $n$-hop transmission assuming symbol rate and modulation level control may be expressed by substituting the expression for the BE of single-hop transmission $f_{1}$ in Eq. (7) into Eq. (8),

$$
f_{n}\left(\gamma, p_{\mathrm{b}}\right)= \begin{cases}2 \beta_{n} n^{\alpha} \gamma / 3 n & 3 / 4 \beta_{n} \leq n^{\alpha} \gamma<3 / \beta_{n} \\ \log _{2}\left(1+\beta_{n} n^{\alpha} \gamma\right) / n & 3 / \beta_{n} \leq n^{\alpha} \gamma \leq 63 / \beta_{n}\end{cases}
$$

where $\beta_{n}=-1.5 / \ln \left(5 p_{\mathrm{b}} / n\right)$.

\subsection{Numerical Results}

Table 1 summarizes the parameters we used in our evaluation. When the number of hops is assumed to be fixed to $n$
Table 1 Parameters used in the evaluation.

\begin{tabular}{c|c}
\hline Parameters & Values \\
\hline Path loss exponent $\alpha$ & $2,3.5$ \\
Required bit error rate $p_{\mathrm{b}}$ & $10^{-3}$ \\
\hline
\end{tabular}

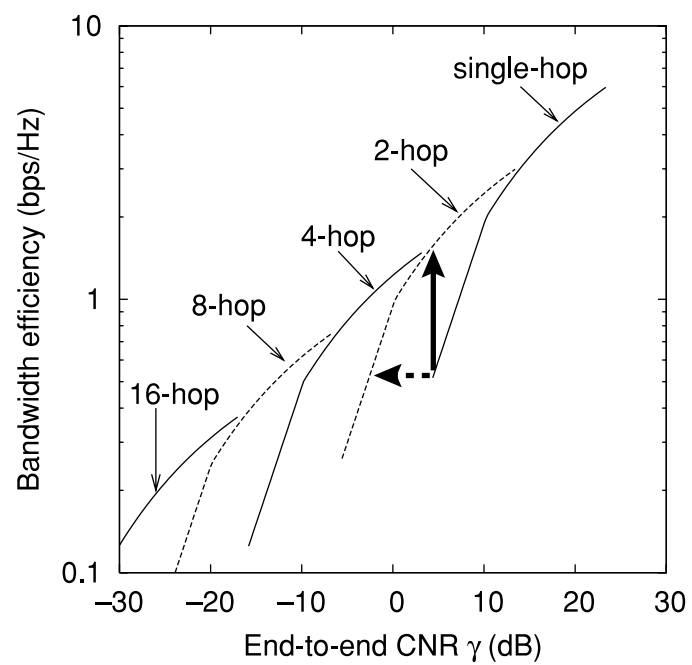

Fig. 3 Dependence of end-to-end throughput of multihop transmission on CNR between source and destination nodes $(\alpha=3.5)$. Bold and dashed arrows correspond to those in Fig. 10(b).

( $n=1,2,4,8,16$ ), the end-to-end CNR dependence of the $\mathrm{BE}(9)$ is as shown in Fig. 3. The figure shows that under low end-to-end CNR conditions introducing multihop transmission may be more effective in terms of the $\mathrm{BE}$; however, the absolute value of the BE may be small. Therefore, symbol rate control and multihop transmission have the same ability to decrease the required end-to-end CNR at the cost of BE.

Next, we consider adaptive route selection by which the route with an optimal number of hops attaining the maximum BE is chosen. Compared to single-hop transmission, the required per-hop BER of $n$-hop transmission is reduced to $1 / n$, and thus the required CNR is increased. Figure 4 shows the optimal number of hops for the different required end-to-end BER $\left(p_{\mathrm{b}}=10^{-3}, 10^{-6}\right)$. Figure 4 also shows the optimal number of hops when Shannon capacity is used. We see that along with the reduction of end-to-end CNR, the amount of difference between the optimal number of hops with symbol rate and modulation level control and that with Shannon capacity increases.

Figure 5 shows the end-to-end CNR dependence of the $\mathrm{BE}$ through the use of adaptive route selection. We see that the difference between the BE with symbol rate and modulation level control and that with Shannon capacity is relatively small. Thus, in this situation, error accumulation does not necessarily become the main factor in capacity degradation.

Thus far, we have assumed the same transmit power irrespective of the number of hops. This assumption provides a fair comparison between single-hop transmission and multihop transmission in terms of energy efficiency because the total transmit energy of all transmitting nodes is the same 


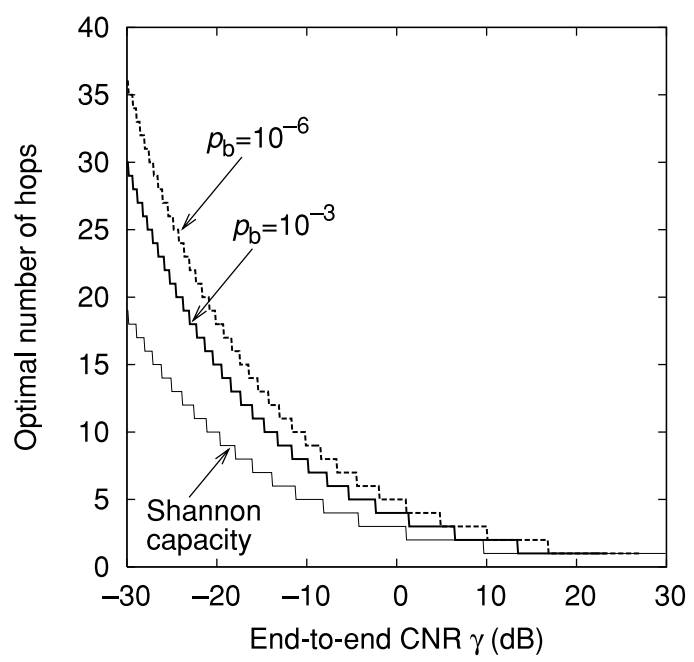

Fig. 4 Effect of accumulated bit error on optimal number of hops ( $\alpha=$ $3.5)$.

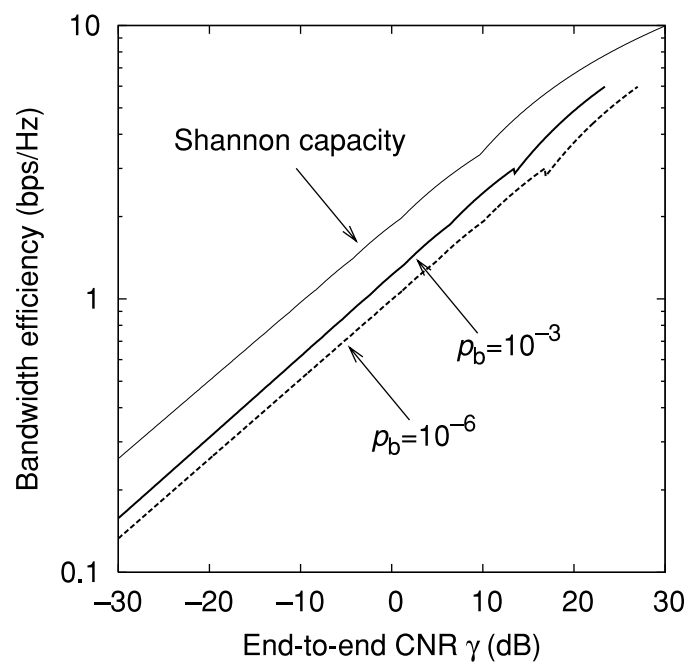

Fig. 5 Effect of accumulated bit error on bandwidth efficiency $(\alpha=3.5)$.

regardless of the number of hops.

Here, we consider the scenario where the transmit power is reduced with the number of hops. The BE of this scenario can be derived from the same Fig. 5 since the end-to-end CNR is increased or decreased in proportion to the transmit power. When the number of hops is optimally selected in terms of the BE, an increase in the end-to-end CNR implies an increase in the BE. Thus, the BE is a monotonically increasing function of the end-to-end CNR or the transmit power per node. Therefore, when the spatial channel reuse is not allowed, transmission at the maximum power is effective from the viewpoint of the BE.

\section{Bandwidth Efficiency of Multihop Transmission with Spatial Channel Reuse}

\subsection{Analysis}

In this section, we investigate in what situation the $\mathrm{BE}$ of multihop transmission may increase through spatial channel reuse. Through the use of symbol rate and modulation level control, the required CINR is adjusted to meet the received CINR. In addition to this, varying the numbers of hops and channels can control the received CINR. Therefore, the numbers of hops and channels are the variables to be optimized.

Until now, we have assumed $n$ different channels for $n$-hop transmission. Now we assume $\ell(<n)$ different channels so that the percentage of time a node can transmit a signal becomes $1 / \ell$. Consider the node receiving the largest interference in a single isolated $n$-hop transmission using $\ell$ channels as shown in Fig. 2(c). Co-channel interference signals come from those nodes separated by the following number of hops, i.e., $\ell-1, \ell+1,2 \ell-1, \ldots,\lceil(\lceil n / \ell\rceil-1) / 2\rceil \ell+$ $(-1)^{\lceil n / \ell\rceil-1}$. Let $C_{n, \ell}$ denote the received power level from the desired node, and let $I_{n, \ell}$ denote the total interference power at the receiving node. Then, the received carrier-tointerference ratio (CIR) $C_{n, \ell} / I_{n, \ell}$ can be written as

$$
\frac{C_{n, \ell}}{I_{n, \ell}}=\left\{\sum_{i=1}^{\lceil n / \ell\rceil-1}\left[\lceil i / 2\rceil \ell+(-1)^{i}\right]^{-\alpha}\right\}^{-1} \equiv \xi .
$$

Let $N$ be the noise power level. The per-hop CNR is then expressed as $C_{n, \ell} / N=n^{\alpha} \gamma$. Therefore, per-hop CINR can be expressed as

$$
\begin{aligned}
\frac{C_{n, \ell}}{N+I_{n, \ell}} & =\frac{1}{\left(C_{n, \ell} / N\right)^{-1}+\left(C_{n, \ell} / I_{n, \ell}\right)^{-1}} \\
& =\left(1 / n^{\alpha} \gamma+1 / \xi\right)^{-1} .
\end{aligned}
$$

Assuming every node adjusts its data rate based on $C_{n, \ell} /(N+$ $\left.I_{n, \ell}\right)$, we get the BE of $n$-hop transmission over $\ell$ channels as

$$
f_{n, \ell}\left(\gamma, p_{\mathrm{b}}\right)=\frac{1}{\ell} f_{1}\left(\left(1 / n^{\alpha} \gamma+1 / \xi\right)^{-1}, p_{\mathrm{b}} / n\right) .
$$

\subsection{Numerical Results}

When we assume the number of hops is fixed to $n$ ( $n=$ $1,2,4,8,16)$ and the number of channels $\ell$ is a variable to be optimized, the end-to-end CNR dependence of the BE (10) is as shown in Fig. 6. The BE can increase through spatial channel reuse under low end-to-end CNR conditions.

Then, we assume the numbers of hops and channels are variables to be chosen optimally in terms of the BE. Figure 7 shows the optimal numbers of hops and channels for different values of the path loss exponent $\alpha$. Multihop transmission outperforms single-hop transmission in terms of the BE below the end-to-end CNR of $9 \mathrm{~dB}$ and $13 \mathrm{~dB}$ for $\alpha=2$ 


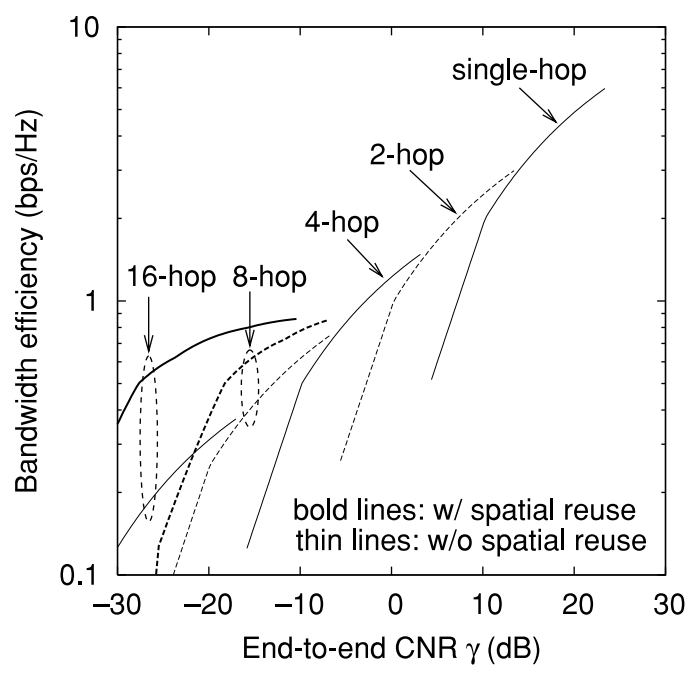

Fig. 6 Bandwidth efficiency with optimal number of channels $(\alpha=3.5)$.

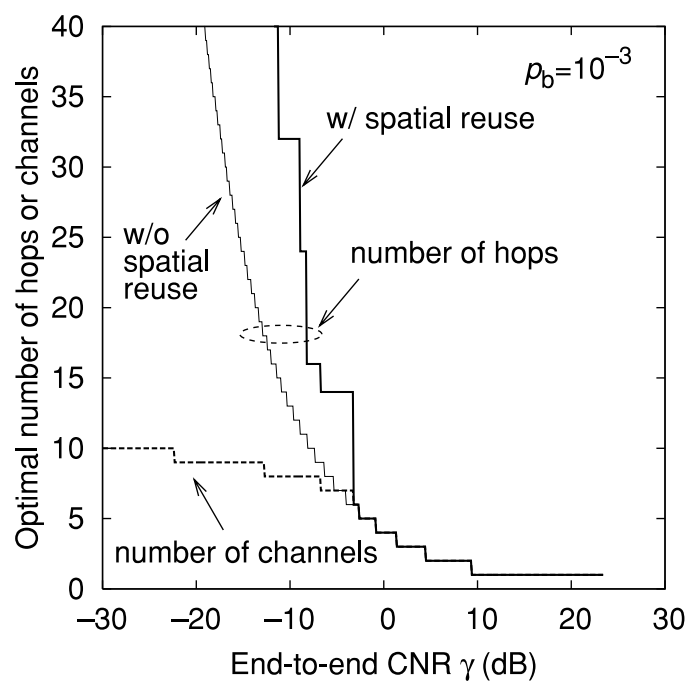

(a) $\alpha=2$.

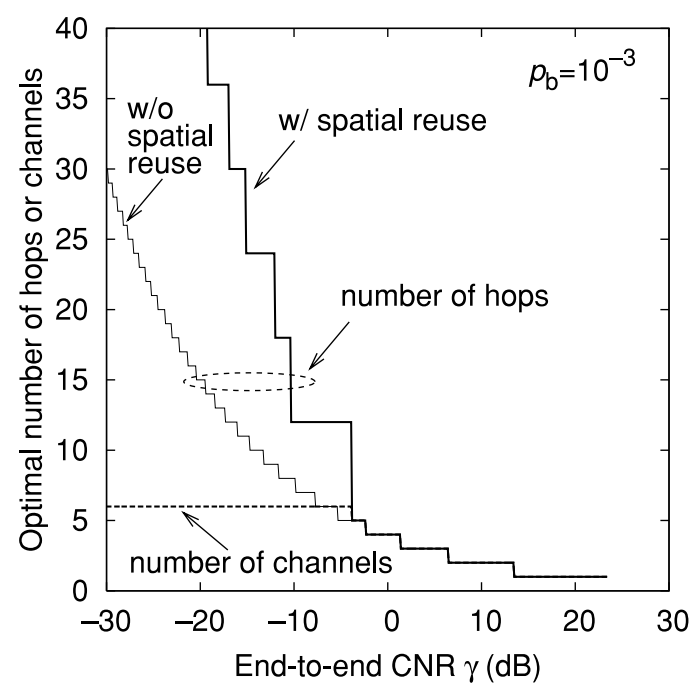

(b) $\alpha=3.5$.

Fig. 7 Optimal numbers of hops and channels for bandwidth efficiency.

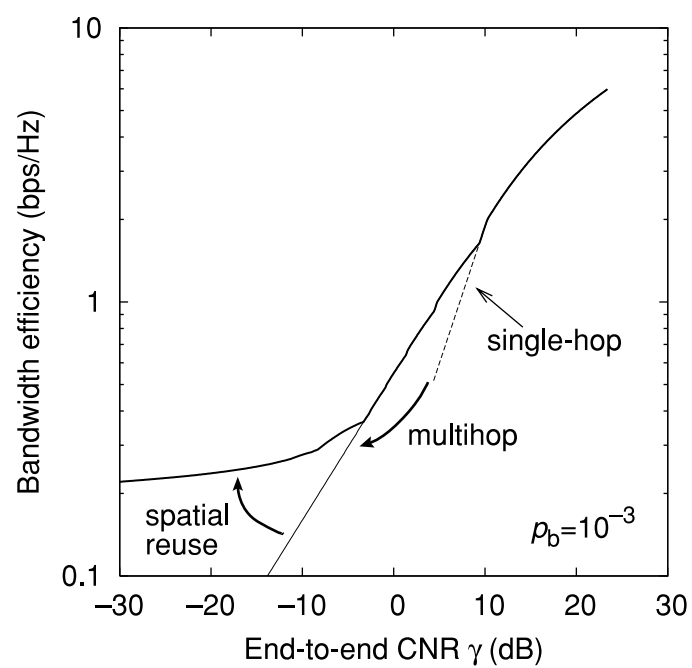

(a) $\alpha=2$.

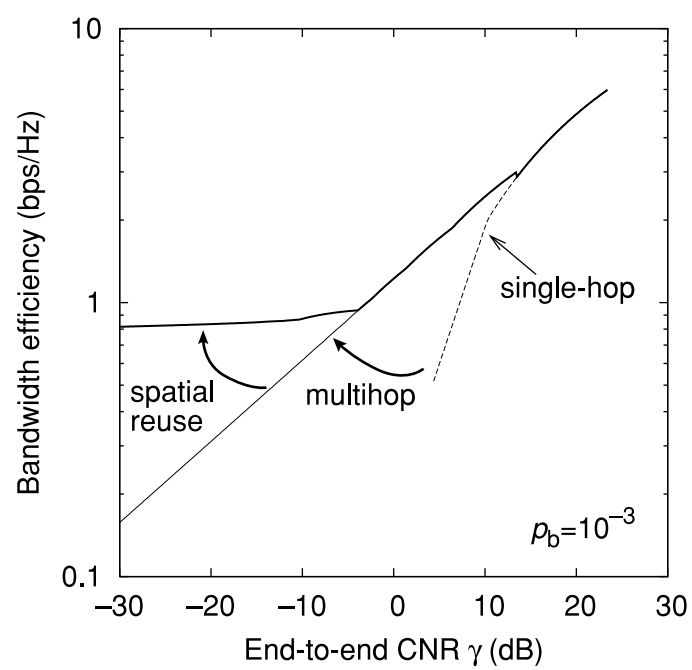

(b) $\alpha=3.5$.

Fig. 8 Bandwidth efficiency with optimal numbers of hops and channels.

and 3.5, respectively. In addition to this, multihop transmission with spatial channel reuse outperforms that without spatial channel reuse below the end-to-end CNR of $-3 \mathrm{~dB}$ and $-4 \mathrm{~dB}$ for $\alpha=2$ and 3.5, respectively. The optimal number of hops increases rapidly with reduction in the end-to-end CNR compared to multihop transmission without spatial channel reuse.

Figure 8 shows the BE with the optimal numbers of hops and channels for different values of the path loss exponent $\alpha$. When the path loss exponent is relatively small, nodes will receive larger interference from simultaneous transmissions. To mitigate the interference, a large number of channels are required, and consequently, the BE will be small.

In contrast to the case without spatial channel reuse, the loss of $\mathrm{BE}$ along with the reduction of end-to-end CNR is relatively small especially when the path loss exponent is large $(\alpha=3.5)$. Therefore, under the condition that the spatial channel reuse is effective, energy efficiency can be 
increased by reducing the transmit power per node at the cost of a slight decrease in the BE.

\section{Area Spectral Efficiency of Multihop Radio Net- works}

In the previous section, we assume that channels are spatially reused in a single isolated multihop route. On the contrary, in this section, we assume that channels are spatially reused only among multihop routes.

We consider the interference-limited situation where co-channel interference totally dominates the thermal noise. Under this environment, the CINR reduces to the CIR. In a single-hop transmission scenario, the decrease of the required CIR allows more simultaneous transmissions in a given area but each node should transmit at a lower rate. In a multihop transmission scenario, the increased number of hops allows more simultaneous transmissions in a given area because adaptive route selection has the same ability as symbol rate control to decrease required end-to-end CINR at the cost of lower end-to-end throughput, as was presented in Sect. 3.2. Therefore, we evaluate the resulting area spectral efficiency (ASE) of multihop radio networks, which is defined as the maximum end-to-end bit rate through multiple hops per unit bandwidth per unit area.

\subsection{Analysis}

The ASE of multihop radio networks is sensitive to lots of factors, particularly fairness, as mentioned in Sect. 1. Since the main purpose of this investigation is to examine the im-

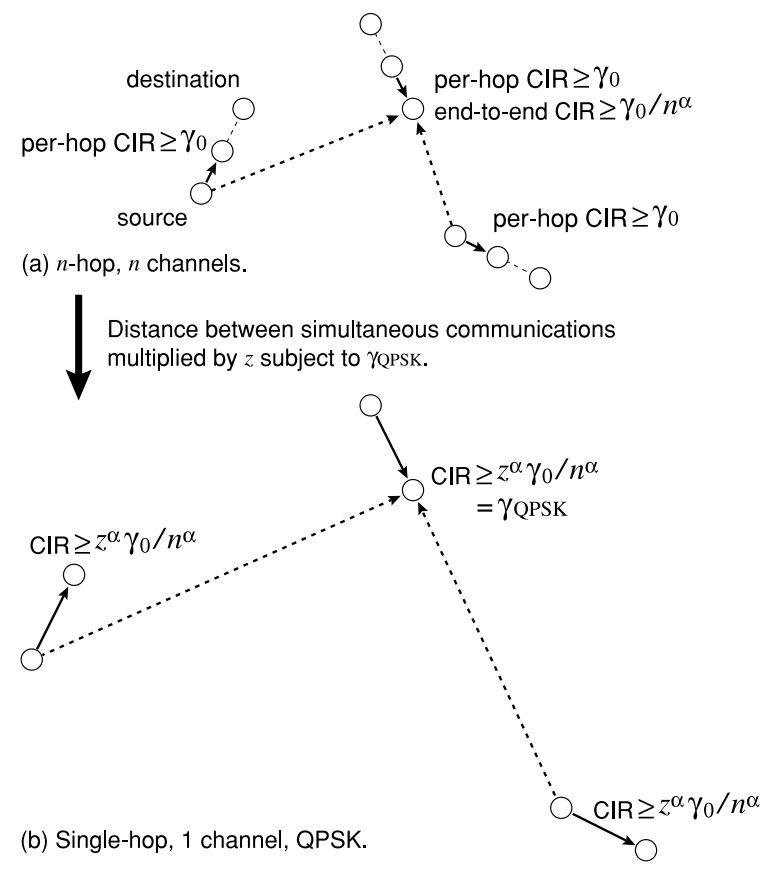

Fig. 9 Models of single-hop and multihop transmissions $(n=2)$. Solid arrows represent desired signals and dashed arrows represent co-channel interference. pact of symbol rate and modulation level control and adaptive route selection on the ASE, we must set aside the fairness problem and investigate the ASE under the condition that the number of hops for all transmissions is the same and the per-hop CIR of all transmissions is greater than a given value $\gamma_{0}$ as shown in Fig. 9(a). In addition to this, we model the topology of $n$-hop transmission as equally spaced $(n-1)$ relaying nodes as in Sect. 3.

Consider the situation where every source node transmits directly to its destination regardless of whether every source-destination pair can communicate directly, and we designate the received CIR as being the end-to-end CIR. Similar to the end-to-end CNR, the end-to-end CIR is considered to be an appropriate parameter to reflect the density of simultaneous communications since the absolute value of this density does not have meaning in our simple model. Therefore, the BE in an interference-limited situation can also be expressed by replacing end-to-end CNR with endto-end CIR in Eq. (9).

Let $t_{n}$ be the BE of $n$-hop transmission. Under the assumption above, the end-to-end CIR of all simultaneous communications are greater than $\gamma_{0} / n^{\alpha}$. We assume that every node adjusts its data rate based on $\gamma_{0}$. Thus, the BE of each $n$-hop transmission may be expressed by using the end-to-end CIR $\gamma_{0} / n^{\alpha}$ as

$$
t_{n}=f_{n}\left(\gamma_{0} / n^{\alpha}, p_{\mathrm{b}}\right) \text {. }
$$

Let $\rho_{n}$ be the density of source-destination pair achieving the given per-hop CIR, $\gamma_{0}$, when transmitting simultaneously. Then, defining the ASE of $n$-hop transmission as

$$
\eta_{n}=t_{n} \cdot \rho_{n}
$$

naturally follows. The goal of this section is to approximate the ASE of multihop radio networks. Through the use of rate adaptation, the required CIR is adjusted to meet the received CIR. In addition to this, changing the distance between simultaneous communications and the number of hops can control the received CIR.

For comparison, we also consider single-hop radio networks with QPSK. We assume the distance between source and destination nodes is the same as that in multihop radio networks. Next, we consider multiplying the distances between simultaneous communications by $z$ subject to the required CIR of QPSK, $\gamma_{\mathrm{QPSK}}$, as shown in Fig. 9(b). The interference power is then reduced to roughly $1 / z^{\alpha}$ of that with $n$-hop transmission, so that the end-to-end CIR of all transmissions satisfy the following condition:

$$
\text { end-to-end } \mathrm{CIR} \geq z^{\alpha} \frac{\gamma_{0}}{n^{\alpha}}=\gamma_{\mathrm{QPSK}} \text {. }
$$

Let $t_{1, \mathrm{QPSK}}=2$ represent the BE; let $\rho_{1, \mathrm{QPSK}}$ be the density of simultaneous communications, and let $\eta_{1, \mathrm{QPSK}}$ be the ASE of single-hop transmission with QPSK. The density of simultaneous communications is reduced to roughly $1 / z^{2}$ of that with $n$-hop transmission

$$
\rho_{1, \mathrm{QPSK}}=\frac{\rho_{n}}{z^{2}}=\frac{\eta_{n}}{z^{2} t_{n}} .
$$


The ASE of single-hop transmission with QPSK is therefore expressed as

$$
\eta_{1, \mathrm{QPSK}}=t_{1, \mathrm{QPSK}} \cdot \rho_{1, \mathrm{QPSK}}=\frac{2 \eta_{n}}{z^{2} t_{n}} .
$$

Substituting Eqs. (4) and (13) into Eq. (11), we get

$$
t_{n}=f_{n}\left(3 / z^{\alpha} \beta_{1}, p_{\mathrm{b}}\right) .
$$

Combining the above equation with Eq. (9) and solving for $z$, we get

$$
z= \begin{cases}n\left(\frac{2}{n t_{n}} \frac{\beta_{n}}{\beta_{1}}\right)^{1 / \alpha} & \frac{1}{2 n} \leq t_{n}<\frac{2}{n} \\ n\left(\frac{3}{2^{n t_{n}}-1} \frac{\beta_{n}}{\beta_{1}}\right)^{1 / \alpha} & \frac{2}{n} \leq t_{n} \leq \frac{6}{n} .\end{cases}
$$

The ASE of $n$-hop transmission is approximated by substituting Eq. (15) into Eq. (14):

$$
\begin{aligned}
\eta_{n} & =\frac{z^{2} t_{n}}{2} \eta_{1, \mathrm{QPSK}} \\
& = \begin{cases}\frac{n^{2} t_{n}}{2}\left(\frac{2}{n t_{n}} \frac{\beta_{n}}{\beta_{1}}\right)^{2 / \alpha} \eta_{1, \mathrm{QPSK}} & \frac{1}{2 n} \leq t_{n}<\frac{2}{n} \\
\frac{n^{2} t_{n}}{2}\left(\frac{3}{2^{n t_{n}}-1} \frac{\beta_{n}}{\beta_{1}}\right)^{2 / \alpha} \eta_{1, \mathrm{QPSK}} & \frac{2}{n} \leq t_{n} \leq \frac{6}{n} .\end{cases}
\end{aligned}
$$

\subsection{Numerical Results}

The combined effect of symbol rate control, modulation level control, and the number of hops on the ASE for the two different path loss exponent $\alpha$ is shown in Fig. 10. The absolute value of $\eta_{1, \text { QPSK }}$ depends on the specific value of the path loss exponent $\alpha$; therefore, there is no way to conduct a direct comparison between Figs. 10(a) and 10(b).

When the nodes are only allowed fixed $n$-hop transmission, the ASE decreases with the increase of the BE from $2 / n$ (QPSK) to $6 / n$ (64QAM). In addition to this, the ASE can decrease with the reduction of the BE from $2 / n$ (QPSK) to $0.5 / n(1 / 4-$ rate QPSK). This result is consistent with other results on multilevel modulation in cellular networks, and it indicates that QPSK rather than 16QAM is the optimal modulation scheme to obtain high ASE [24].

When the nodes are allowed to select the number of hops, as expected, the ASE increases with the number of hops at the cost of BE. Recall the results in Fig. 3 where symbol rate control and adaptive route selection have the same tradeoff between the BE and the end-to-end CNR. However, only adaptive route selection has a tradeoff between the ASE and the BE. This result is also consistent with other results reported in investigations of cellular networks that show a tradeoff between the ASE and the BE of single-hop transmission [4].

By comparing Figs. 3 and 10(b), one can see that increase of the $\mathrm{BE}$ resulting from varying the number of hops

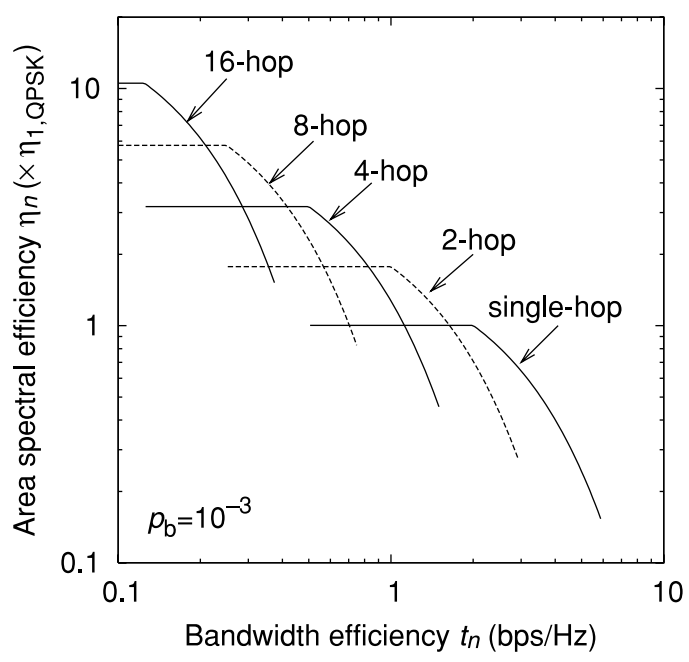

(a) $\alpha=2$.

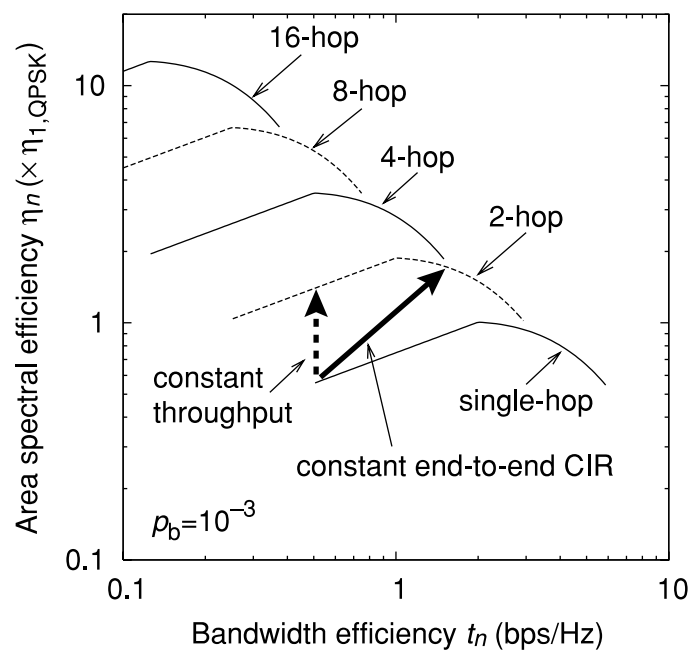

(b) $\alpha=3.5$.

Fig. 10 Area spectral efficiency $\eta_{n}$ vs. bandwidth efficiency $t_{n}$. Bold and dashed arrows correspond to those in Fig. 3.

(as shown by the bold arrow in Fig. 3) corresponds to the enhancement of the ASE by fixing the density of simultaneous communications (as shown by the bold arrow in Fig. 10(b)). One can also see that decreasing the required end-to-end CIR through the use of multihop transmission (as shown by the dashed arrow in Fig. 3) corresponds to the enhancement of the ASE by varying the number of hops and the density of simultaneous communications and fixing the BE (as shown by the dashed arrow in Fig. 10(b)).

\section{Concluding Remarks}

Assuming symbol rate and modulation level control, we have considered two types of spectral efficiency for multihop radio networks. First, we examined the spectral efficiency of a single isolated multihop route, named as bandwidth efficiency, which is defined as the maximum end-toend bit rate through multiple hops per unit bandwidth. We have taken into account the effect of bit error accumulation 
over multihop transmission. We found a relationship between the bandwidth efficiency of $n$-hop transmission and that of single-hop transmission. Numerical results revealed that symbol rate control and adaptive route selection have the same ability to enhance the end-to-end communication range at a loss of bandwidth efficiency.

Next, we also investigated the overall spectral efficiency of multihop radio networks, named as area spectral efficiency, which is defined as the maximum end-to-end bit rate through multiple hops per unit bandwidth per unit area. We derived an expression for area spectral efficiency as a function of end-to-end throughput and the number of hops. Numerical results revealed a significant difference between symbol rate control and adaptive route selection. The use of symbol rate control can decrease area spectral efficiency. On the other hand, a tradeoff between the area spectral efficiency and the end-to-end throughput of multihop transmission is such that the use of multihop transmission can increase the area spectral efficiency. Note that these results are consistent with those of other research on the area spectral efficiency of cellular networks with modulation level control.

We would like to emphasize that the purpose of this paper is to investigate and clarify the circumstances under which multihop transmission provides superior performance over that of single-hop transmission. We hope the results presented in this paper will provide insights useful in designing multihop radio networks.

\section{Acknowledgments}

We greatly appreciate the benefits received in fruitful discussions with Dr. N. Umeda and Mr. A. Fujiwara of the Wireless Laboratories, NTT DoCoMo Inc. This work is supported in part by a Grant-in-Aid from the 21st Century COE Program (no. 14213201) of the Ministry of Education, Culture, Sports, Science and Technology (MEXT), Japan, a Grant-in-Aid for Scientific Research (A) (no. 16206040) from the Japan Society for the Promotion of Science (JSPS), and the JSPS Research Fellowships for Young Scientists (no. 16001178).

\section{References}

[1] J. Weatherall and A. Jones, "Ubiquitous networks and their applications," IEEE Wirel. Commun. Mag., vol.9, no.1, pp.18-29, Feb. 2002.

[2] R. Ramanathan and J. Redi, "A brief overview of ad hoc networks: Challenges and directions," IEEE Commun. Mag., vol.40, no.5, pp.20-22, May 2002.

[3] K. Mase, M. Sengoku, and S. Shinoda, "A perspective on nextgeneration ad hoc networks-A proposal for an open community network," IEICE Trans. Fundamentals, vol.E84-A, no.1, pp.98-106, Jan. 2001.

[4] R. Haas and J.C. Belfiore, "Spectrum efficiency limits in mobile cellular systems," IEEE Trans. Veh. Technol., vol.45, no.1, pp.33-40, Feb. 1996.

[5] M.S. Alouini and A.J. Goldsmith, "Area spectral efficiency of cellular mobile radio systems," IEEE Trans. Veh. Technol., vol.48, no.4, pp.1047-1066, July 1999.
[6] K.J. Hole and G.E. Øien, "Spectral efficiency of adaptive coded modulation urban microcellular networks," IEEE Trans. Veh. Technol., vol.50, no.1, pp.205-222, Jan. 2001.

[7] T. Mukai, H. Murata, and S. Yoshida, "Study on channel selection algorithm and number of established routes of multi-hop autonomous distributed radio networks," IEICE Trans. Commun. (Japanese Edition), vol.J85-B, no.12, pp.2080-2086, Dec. 2002.

[8] J. Jun and M.L. Sichitiu, "Fairness and QoS in multihop wireless networks," Proc. IEEE VTC 2003-Fall, pp.6-9, Oct. 2003.

[9] H. Kwak and S. Yoshida, "Tradeoff between fairness and throughput in multi-hop wireless LAN," IEICE Trans. Fundamentals, vol.E87A, no.7, pp.1733-1741, July 2004.

[10] V. Gambiroza, B. Sadeghi, and E.W. Knightly, "End-to-end performance and fairness in multihop wireless backhaul networks," Proc. MOBICOM 2004, pp.287-301, Sept.-Oct. 2004.

[11] M. Grossglauser and D.N.C. Tse, "Mobility increases the capacity of ad hoc wireless networks," IEEE/ACM Trans. Netw., vol.10, no.4 pp.477-486, Aug. 2002.

[12] K. Yamamoto and S. Yoshida, "Analysis of distributed route selection scheme in wireless ad hoc networks," Proc. IEEE PIMRC '04, Sept. 2004

[13] K. Yamamoto and S. Yoshida, "Game-theoretic approach to capacity and stability evaluations of decentralized adaptive route selections in wireless ad hoc networks," IEICE Trans. Commun., vol.E88-B, no.3, pp.1009-1016, March 2005.

[14] P. Gupta and P.R. Kumar, "The capacity of wireless networks," IEEE Trans. Inf. Theory, vol.46, no.2, pp.388-404, March 2000.

[15] S. Toumpis and A.J. Goldsmith, "Capacity regions for wireless ad hoc networks," IEEE Trans. Wireless Commun., vol.2, no.4, pp.736-748, July 2003.

[16] S. Toumpis and A.J. Goldsmith, "Performance, optimization, and cross-layer design of media access protocols for wireless ad hoc networks,” Proc. IEEE ICC ’03, pp.2234-2240, May 2003.

[17] M. Sikora, J.N. Laneman, M. Haenggi, D.J. Costello, and T. Fuja "On the optimum number of hops in linear wireless networks," IEEE Information Theory Workshop, pp.165-169, Oct. 2004.

[18] M. Haenggi, "Twelve reasons not to route over many short hops," Proc. IEEE VTC 2004-Fall, Sept. 2004.

[19] A.J. Goldsmith and S.G. Chua, "Variable-rate variable-power MQAM for fading channels," IEEE Trans. Commun., vol.45, no.10, pp.1218-1230, Oct. 1997.

[20] T. Ue, S. Sampei, and N. Morinaga, "Symbol rate controlled adaptive modulation/TDMA/TDD for wireless personal communication systems," IEICE Trans. Commun., vol.E78-B, no.8, pp.1117-1124, Aug. 1995.

[21] S. Sampei, Applications of Digital Wireless Technologies to Global Wireless Communications, Prentice-Hall, 1997.

[22] P. Jung, P.W. Baier, and A. Steil, "Advantages of CDMA and spread spectrum techniques over FDMA and TDMA in cellular mobile radio applications," IEEE Trans. Veh. Technol., vol.42, no.3, pp.357364, Aug. 1993.

[23] A.J. Goldsmith and S.B. Wicker, "Design challenges for energyconstrained ad hoc wireless networks," IEEE Wireless Commun. Mag., vol.9, no.4, pp.8-27, Aug. 2002.

[24] N. Morinaga, M. Yokoyama, and S. Sampei, "Intelligent radio communication techniques for advanced wireless communications systems," IEICE Trans. Commun., vol.E79-B, no.3, pp.214-221, March 1996. 


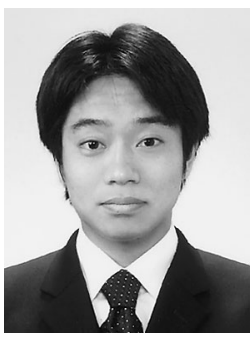

Koji Yamamoto was born in Kochi, Japan, on May 2, 1979. He received a B.E. degree in electrical and electronic engineering and an M.E. degree in informatics from Kyoto University in 2002 and 2004, respectively. He is currently a research fellow of the Japan Society for the Promotion of Science (JSPS), studying toward his Ph.D. degree at the Graduate School of Informatics, Kyoto University. His research interests include next-generation wireless communication systems and wireless ad hoc networks.

He received the IEEE VTS Japan 2003 Young Researcher's Encouragement Award and the PIMRC 2004 Best Student Paper Award. He is a student member of the IEEE.

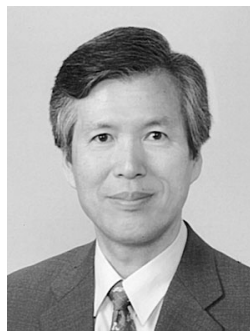

Susumu Yoshida received the B.E., M.E. and $\mathrm{Ph} . \mathrm{D}$. degrees in electrical engineering from Kyoto University, Kyoto, Japan in 1971, 1973 and 1978, respectively. Since 1973, he has been with the Faculty of Engineering, Kyoto University and currently he is a full professor of the Graduate School of Informatics, Kyoto University. During the last 20 years, he has been mainly engaged in the research of wireless personal communications. His current research interest includes wireless transmission technologies beyond IMT-2000 and wireless ad hoc networks. During 1990-1991, he was a visiting scholar at WINLAB, Rutgers University, U.S.A. and Carleton University in Canada. He served as an Executive Committee Chairperson of PIMRC'99, Osaka and also as a Technical Program Committee Chairperson of IEEE VTC 2000-Spring, Tokyo. He was a guest editor of IEEE J-SAC on Wireless Local Communications published in April and May 1996. He was a Director, Journal and Transactions of IEICE during 2002-2004. He was awarded the Young Researcher's Award in 1978 and the Achievement Award in 1993 both from the IEICE. 